\title{
Diagnostic Accuracy and Therapeutic Efficacy of Digital Single-Operator Cholangioscopy for Biliary Lesions and Stenosis
}

\author{
Kilian Weigand Merlin Küchle Ina Zuber-Jerger Martina Müller \\ Arne Kandulski \\ Department of Internal Medicine I, University Hospital Regensburg, Regensburg, Germany
}

\section{Keywords}

Cholangioscopy · Digital single-operator cholangioscopy ·

Endoscopic retrograde cholangiopancreatography · Biliary lesion - Biliary stricture

\begin{abstract}
Background/Aims: Digital single-operator cholangioscopy (dSOC) has revolutionized bile duct visualization. Interventions like electrohydraulic or laser lithotripsy, inspection of suspicious areas, and targeted biopsies have become possible quick and easy. One main indication for dSOC remains the evaluation of indeterminate biliary strictures. Objective and Methods: We analyzed 180 consecutive dSOCs procedures performed in a high-volume tertiary center to evaluate sensitivity, specificity as well as positive and negative predictive values (PPV and NPV) for indeterminate strictures. Furthermore, technical success and complications were analyzed. Results: In 92-97\%, the region of interest was reached and successfully visualized. In $83-100 \%$, targeted biopsies were obtained from the suspicious area. Only the distal bile duct was less successful with only 84 and $62 \%$, respectively. In general, dSOC procedures were safe. Cholangitis was the main complication. Regarding the diagnostic accuracy of dSOC of indeterminate biliary strictures, we found a sensitivity of 0.87 and specificity of 0.88 , over all. Within the whole
\end{abstract}

karger@karger.com www.karger.com/dig

Karger $\stackrel{\text { ' }}{5}$

GOPEN ACCESS
(C) 2021 The Author(s)

Published by S. Karger AG, Basel

This is an Open Access article licensed under the Creative Commons Attribution-NonCommercial-4.0 International License (CC BY-NC) (http://www.karger.com/Services/OpenAccessLicense), applicable to the online version of the article only. Usage and distribution for commercial purposes requires written permission. cohort, the investigators' assessment directly after dSOC had a PPV of 0.63 and a NPV of 0.97 . In patients with biliary lesions or stenosis suspicious for malignancy, the dSOC-based visual diagnosis revealed a very high diagnostic accuracy with sensitivity and specificity of $1.0(95 \% \mathrm{Cl} 0.86-1.0)$ and 0.76 (95\% Cl 0.56-0.9) with a PPV of $0.77(95 \% \mathrm{Cl} 0.59-0.9)$ and a high NPV of 1.0 ( $95 \% \mathrm{Cl} 0.85-1.0)$. Conclusions: Our study demonstrates that $\mathrm{dSOC}$ has a high diagnostic accuracy as well as a favorable safety profile. Therefore, dSOC should be discussed as standard of care during endoscopic retrograde cholangiography for indeterminate biliary lesions.

(c) 2021 The Author(s)

Published by S. Karger AG, Basel

\section{Introduction}

Endoscopic retrograde cholangiopancreatography (ERCP) as a standard endoscopic intervention for biliary diseases has been established for decades. Main indications are indeterminate biliary lesions with suspected malignancy, strictures, bile duct stones, cholangitis, and postsurgical complications like strictures and leakage [16]. Due to the two-dimensional imaging in fluoroscopy, ERCP has also distinct limitations, especially for the characterization of biliary strictures and the differentiation between inflammatory, malignant, or fibrotic pathogen- 
esis. In addition, tissue sampling by ERCP-directed brushing has a low sensitivity and specificity $[7,8]$. Furthermore, guidewire placement in complicated strictures, malignant stenosis, or after surgery can be difficult only using fluoroscopy guidance.

Transpapillar cholangioscopy allows direct visualization of the biliary system up to biliary branches of second and third order. 2006 the SpyGlass ${ }^{\mathrm{TM}}$ single-operator cholangioscopy (SOC) (Boston Scientific Coro., Natick, MA, USA) has been introduced, making cholangioscopy widely available and feasible $[9,10]$. While the first generation of SOC was quite low in visualization quality due to a fiber optical probe, the introduction of the digital high-resolution cholangioscopy SpyGlass DS ${ }^{\mathrm{TM}}$ (dSOCS) (Boston Scientific Coro., Natick, MA, USA) in 2015 has achieved much better biliary endoscopic imaging quality. Over the last years, some studies have demonstrated safety profile and technical accuracy [11-13]. Especially, the diagnostic efficacy, sensitivity, and specificity for indeterminate biliary lesions have been investigated in different studies $[8,14,15]$. These studies included tissue sampling under direct visualization control through the SpyGlass $\mathrm{DS}^{\mathrm{TM}}$ using a small biopsy forceps. Therapeutic SOC has been further evaluated for the treatment of difficult biliary stones using electrohydraulic lithotripsy (EHL) or laser lithotripsy [16] and for management of complex biliary strictures $[15,17,18]$.

We performed a single-center study to analyze dSOCs using SpyGlass DS ${ }^{\mathrm{TM}}$ at a high-volume endoscopic center at a university hospital specialized for hepatology, gastrointestinal oncology, digestive and liver surgery, including liver transplantation. We performed a retrospective analysis of all dSOC procedures that have been performed from 2016 till 2017 for any reason, to evaluate the accuracy of the cholangioscopic image-based diagnosis for the prediction of malignancy in strictures and suspect biliary lesions.

Furthermore, therapeutic efficacy of cholangioscopicassisted guidewire placement, stone destruction, and stricture therapy was examined. In addition, technical efficacy, safety, and complications were evaluated.

\section{Patients and Methods}

\section{Study Design}

This retrospective study was performed at the Department of Internal Medicine I at the University Hospital Regensburg. This study was approved by the local Ethics Committee of the University of Regensburg (No. 18-1121-104) and was performed according the updated guidelines of good clinical practice and updated Declaration of Helsinki. Prior to the cholangioscopy informed consent for the examination was obtained from all patients.
All patients who underwent dSOC using the SpyGlass DS ${ }^{\mathrm{TM}}$ system at the University Hospital Regensburg between April 2016 and December 2017 were included in a clinical database. Additional clinical data and the clinical follow-up until August 2018 were retrieved from the clinical data information system. Patients with suspicious biliary lesions were routinely followed up based on their specific findings (biopsies with malignant histology, surgery, radiological imaging, or endoscopic follow-up including repetitive dSOC). The minimum follow-up period in the study cohort was at least 90 days.

\section{Technical Aspects and Definition of Study Outcome}

\section{Parameters}

All examinations were performed by 3 highly experienced investigators with ERC cases $>200 /$ year. Endoscopic papillotomy was performed prior dSOCs or had been done in a previous investigation. Aspiration of biliary fluid for microbial analysis was performed routinely after guidewire insertion and before massive contrast injection.

Papillary intubation and insertion of the cholangioscope into the common bile duct (CBD) were achieved directly or by guidewire assistance. In general, cholangioscopy was performed before massive contrast injection or any other procedure in the bile ducts to reduce artificial findings. In all cases, the SpyGlass DS ${ }^{\mathrm{TM}}$ device was deeply introduced into the biliary system as far as possible, at least proximal of the bifurcation. Careful examination of the biliary mucosa and potential indeterminate strictures was performed during retraction of the dSOC device. Strictures were visualized and described similar to the recently introduced classification system for macroscopic classification of neoplastic lesions [19]. Macroscopic criteria for neoplastic lesions have been introduced criteria for potential malignant lesions are irregular vascular patterns, ulcerations, polypoid appearance, and irregular surface with/without spontaneous bleeding.

All examinations were performed under $\mathrm{CO}_{2}$ insufflation. Prophylactic antibiotic treatment was not performed routinely for each examination but was applied for other indications such as dilation therapy, lithotripsy, local ablation, or incomplete drainage of distinct parts of the biliary tract. In case of preexisting cholangitis or other infection, most patients already received antibiotic therapy before.

The diagnostic accuracy of the visual diagnosis of biliary lesions or stenosis by dSOCs using the SpyGlass DSTM system was evaluated. According to the cholangioscopic imaging, each biliary lesion was diagnosed as a potential malignant stenosis or nonmalignant stenosis in the written report of each investigator. Biopsies ( $>6$ biopsies per lesion and single biopsy technique) were taken from the stenosis or strictures under visual guidance using the SpyBite forceps (SpyBite, single-use, and $1.0 \mathrm{~mm}$ diameter) and directly rinsed into formalin for pathological examination. Successful biopsy was defined as true-positive or true-negative histology.

The diagnostic accuracy (sensitivity, specificity, positive, and negative predictive values [PPV and NPV]) of the visual cholangioscopic diagnosis was analyzed according to histopathological results, surgical outcomes in case of resection, or the clinical history in case of clinical follow-up (including endoscopic follow-up and follow-up imaging by CT scan or MRI).

In case of strictures that had not been passed by guidewire before, guidewire placement was achieved under visual control. Therapeutic success in case of complicated strictures or stenosis was defined by successful guidewire placement across the stricture 
Table 1. Patients characteristics are shown

\begin{tabular}{lc}
\hline Characteristics & \\
\hline Patients, $n$ & 117 \\
Males, \% & 66 \\
Females, \% & 34 \\
Cholangioscopies, $n$ & $180^{*}$ \\
Average age, years & 64 \\
Maximum age, years & 97 \\
Minimum age, years & 7 \\
\hline
\end{tabular}

*Percutaneous access via PTC in 4 cases.
Table 2. Indications for the cholangioscopies performed in this study

\begin{tabular}{ll}
\hline Indication & Patients, $n$ \\
\hline Suspicion of biliary malignancy & 37 \\
Indeterminate stenosis with cholestasis & 33 \\
PSC/SSC & 32 \\
Biliary stones & 34 \\
Control after biliary intervention/follow-up & 34 \\
Others & 10
\end{tabular}

PSC, primary sclerosing cholangitis; SSC, secondary sclerosing cholangitis. allowing endoscopic therapy such as balloon dilation or stent placement.

For EHL, the Northgate Autolith iEHL generator with a $1.9 \mathrm{~F}$ probe or the Walz EHL probe (P2,4/3000/f probe; Walz Elektronik $\mathrm{GmbH}$, Rohrdorf, Germany) was used. After fragmentation, stone clearance was achieved by using a basket or balloon catheter. In case of incomplete clearance, a plastic stent was inserted until the procedure was repeated.

The technical success of dSOC in our study was defined

- As the successful placement of the guidewire across the biliary stricture allowing subsequent balloon dilation and/or endoprosthesis placement;

- As the successful identification of a suspicious and previous defined region of interest (ROI) with cholangioscopic-guided biopsies;

- Successful destruction of intraluminal stones using EHL. dSOC-associated complications were defined and extracted from the clinical data sheets and clinical information system. The following complications were defined (I) bleeding: significant drop of hemoglobin, hematochezia, and need for endoscopy; (II) cholangitis: new onset of fever, significant increase of inflammatory markers and liver enzymes, requiring antibiotic therapy; (III) pancreatitis: new onset of abdominal pain and a three-fold increase in serum lipase within $24-48 \mathrm{~h}$ after the intervention; (IV) perforation. No embolism occurred in our study cohort.

\section{Statistical Analysis}

Descriptive statistics including means, standard error of mean, and frequencies were calculated. Sensitivity, specificity, PPV, $\mathrm{NPV}$, and accuracy were calculated for visual, cholangioscopic diagnosis of suspected lesions. Data analysis was performed using Graphpad Prism 6.0 (GraphPad Software; La Jolla, CA, USA).

\section{Results}

\section{Patients' Characteristics and Procedural}

Complications

180 investigations with dSOCs in 117 patients (mean age 64 years, range 7-97 years, male 66\%, and female
$34 \%$ ) were included in the analysis (see Table 1). In 4 patients, dSOCs was performed by percutaneous biliary access after percutaneous transhepatic biliary drainage.

Indications for dSOCs were retrieved retrospectively from the clinical information system (see Table 2). Most frequent indications were (a) indeterminate biliary strictures with suspected malignancy, (b) other stenosis, for example, postsurgical, (c) patients with primary sclerosing cholangitis or secondary sclerosing cholangitis, or (d) biliary stones (see Table 2).

During the study period, especially in patients with sclerosing cholangitis, cholangioscopy was performed more than once if risk of malignancy was considered high. Further indications for a second examination during the study period were controlled after lithotripsy to rule out residual stones and control of indeterminate stricture with negative histology to take biopsies again when there was still suspicion of cancer.

In $86.1 \%$ of dSOCs investigations (155/180 investigations), no complications occurred. Most frequent complication is post-dSOCs cholangitis (10\%; 18/180 cases), followed by intraductal bleeding $(2.8 \% ; 5 / 180$ cases $)$. For further details, see Table 3.

\section{Accessibility of Targeted Structures and Technical} Success of Digital Single-Operator Cholangioscopies

As ROI to be reached during dSOC, we defined 3 parts of the CBD (distal, mid, and proximal) as well as the intrahepatic biliary system. Accessibility of the ROI was excellent for the mid, proximal CBD, and the intrahepatic biliary system, whereas ROIs in the distal CBD were successfully reached only in $84 \%$ of the cases. Also, biopsies were difficult to take in the distal $\mathrm{CBD}$, mainly due to the instable position of the cholangioscope in the distal CBD (see Table 4). 
Table 3. Complications that occurred during or after the cholangioscopy are shown in total numbers (and percent) of the performed investigations

\begin{tabular}{lc}
\hline Complications & Investigations, $n(\%)$ \\
\hline None & $155(86.1)$ \\
Cholangitis & $18(10.0)$ \\
Bleeding & $5(2.8)$ \\
Perforation & $1(0.6)$ \\
Pancreatitis & $1(0.6)$ \\
\hline
\end{tabular}

In addition, Table 4 shows the performance of EHL or cholangioscopic-guided guidewire insertion of a defined intrahepatic bile duct. dSOC-guided guidewire placement was performed when conventional ERC and deep cannulation failed with at least 2 different wires (straight and bend) within $15 \mathrm{~min}$. Successful bile duct stone treatment was defined as complete bile duct clearance during the first intervention.

\section{Diagnostic Accuracy for Digital Single-Operator Cholangioscopy-Related Diagnosis for Suspicious Biliary Lesions}

All patients with already known biliary malignancy (19 patients) were excluded from the final analysis. Retrospectively, we categorized all patients based on the predSOCs clinical suspicion for malignancy: (I) no clinical "a-priori" suspicion (67\%; 108/161 of the cases); (II) clinical or radiological "a-priori" uncertainty $(21 \%$; $34 / 161$ of the cases); and (III) high "a-priori" suspicion for malignancy $(12 \%, 19 / 161$ of the cases). These data as well as the assessment by the investigator based on the visualization are shown in Figure 1. The categorization was done using the report of the investigator of the initial dSOC only to avoid bias as good as possible in this retrospective analysis.

By dSOCs, we described 43 suspicious lesions. Malignancy was confirmed in 27 of those cases either by cholangioscopic-guided biopsies, by surgical outcome, or during follow-up (Re-dSOC; MRI/CT; surgery). On the other hand, 118 cholangioscopic unsuspicious findings were reported, in whom only 4 cases turned out to be malignant in the follow-up. Based on that retrospective data, we calculated a sensitivity of 0.87 (95\% CI $0.70-0.96)$ and specificity 0.88 (95\% CI $0.81-0.93$ ) as well as a PPV of 0.63 (95\% CI 0.47-0.77) and a NPV of 0.97 (95\% CI 0.92-0.99) for the whole cohort.

Diagnostic Accuracy and Efficacy of dSOC
Table 4. Shown is the ROI that was to be investigated during cholangioscopy and in how many cases it was reached and visualized

\begin{tabular}{|c|c|c|c|}
\hline & $\begin{array}{l}\text { ROI, } \\
n / N(\%)\end{array}$ & $\begin{array}{l}\text { ROI reached, } \\
n / N(\%)\end{array}$ & $\begin{array}{l}\text { Successful } \\
\text { biopsy if ROI } \\
\text { was suspicious, } \\
n / N(\%)\end{array}$ \\
\hline Distal CBD & $44 / 180(24)$ & $37 / 44(84)$ & $8 / 13(62)$ \\
\hline Mid-CBD & $44 / 180(24)$ & $41 / 44(93)$ & $5 / 6(83)$ \\
\hline Proximal CBD & $77 / 180(43)$ & 75/77 (97) & $20 / 21(95)$ \\
\hline Intrahepatic bile system & $13 / 180(7)$ & $12 / 13(92)$ & $1 / 1(100)$ \\
\hline Hepaticojejunostomy & $4 / 180(1)$ & $3 / 4(75)$ & $2 / 2(100)$ \\
\hline Intervention & & $\begin{array}{l}\text { Procedures, } \\
n / N(\%)\end{array}$ & $\begin{array}{l}\text { Successful } \\
\text { intervention, } \\
n / N(\%)\end{array}$ \\
\hline EHL & & $24 / 180(13)$ & $20 / 24(83)$ \\
\hline Guidewire insertion & & $31 / 180(17)$ & $27 / 31(87)$ \\
\hline
\end{tabular}

The last row shows in how many cases the region looked suspicious and in how many cases biopsy could be performed. In the lower part of the table, the performed interventions during cholangioscopy and success rates of the procedure are listed. In case of EHL, complete stone clearance during the first intervention is meant. CBD, common bile duct; EHL, electrohydraulic lithotripsy; ROI, region of interest.

We also calculated sensitivity, specificity, PPV, and NPV in the predefined clinical subgroups (see Fig. 1; [I] "no clinical a-priori suspicion"; [II] clinical or radiological "a-priori uncertainty"; [III] "high a-priori suspicion" for malignancy).

For patients with "low or no clinical a-priori suspicion," (I) sensitivity and specificity were $0.5(0.22-0.78)$ and $0.92(0.85-0.96)$ with PPV of $0.34(0.14-0.61)$ but NPV of 0.96 (0.90-0.98). In contrast, for patient with "a high a-priori suspicion" for malignancy $(n=19)$, sensitivity was $1.0(0.82-1.00)$, specificity $1.0(0.05-1.00)$ as well as PPV of $1.0(0.82-1.00)$ and NPV of $1.0(0.05-1.0)$ were high. In patients with "a-priori uncertainty" of indeterminate biliary strictures $(n=34)$, sensitivity was $1.0(0.61-$ $1.00)$, specificity $0.75(0.57-0.87)$, PPV $0.46(0.23-0.71)$, and a high NPV of $1.0(0.85-1.00)$ for the visual diagnosis of biliary malignancy. For all patients $(n=53)$ with any kind of biliary lesion with suspicion for malignancy ("high a-priori suspicion" + "a-priori uncertainty"), sensitivity was 1.0 (95\% CI 0.86-1.0), specificity 0.76 (95\% CI 0.56-0.9) with a calculated PPV 0.77 (95\% CI 0.59-0.9), and a high NPV of 1.0 (95\% CI $0.85-1.0)$ for the visual diagnosis of biliary malignancy. 


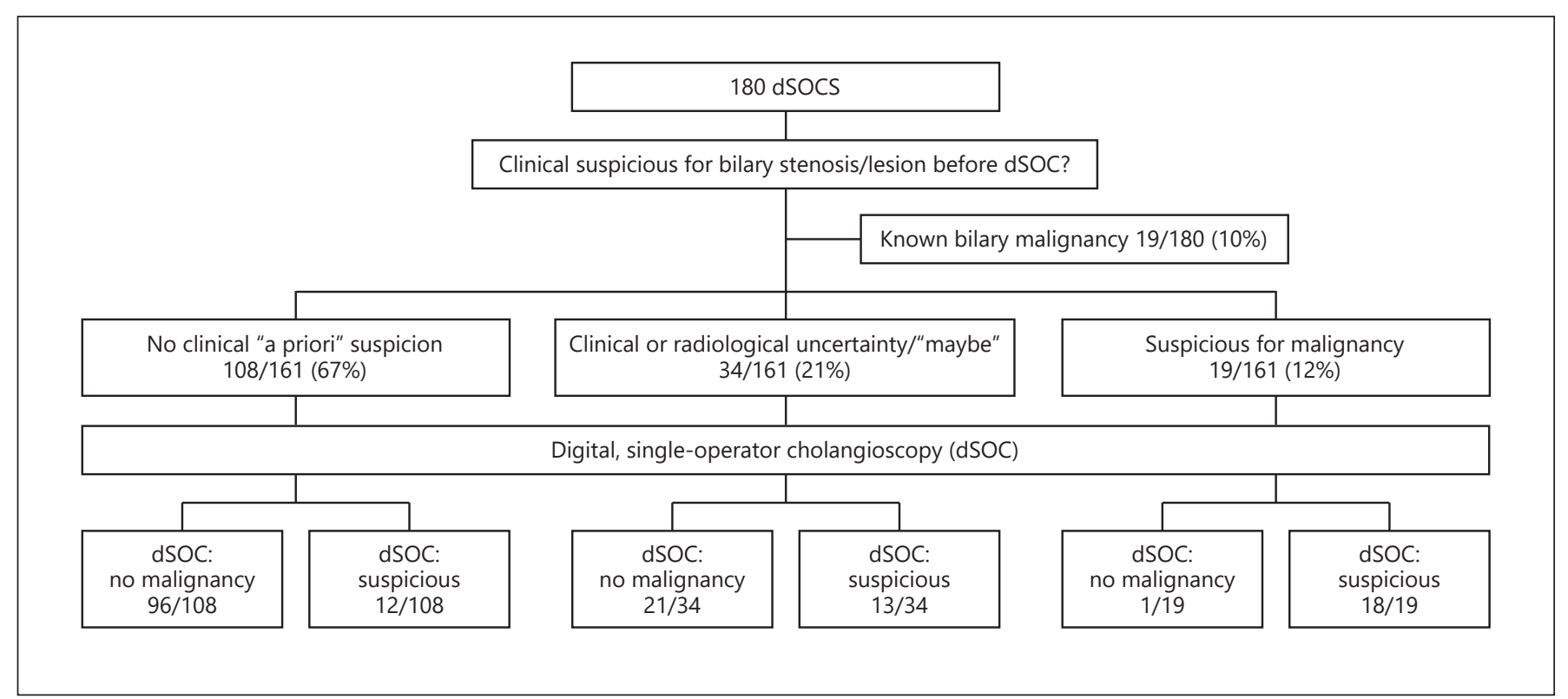

Fig. 1. Shown are the performed dSOCs of this study, the clinically suspected finding before cholangioscopy as well as the assessment of the investigator after dSOC based on the visualized impression. dSOC, digital singleoperator cholangioscopy.

\section{Discussion}

dSOC has already become standard of care and complementary to ERC for biliary interventions, at least in specialized high-volume centers. For difficult biliary stones, therapeutic dSOC combined with EHL is the most effective endoscopic therapy $[11,16]$. dSOCs have been demonstrated to be a safe procedure, also in elderly patients. Diagnostic accuracy of dSOC-directed biopsies is higher than brush cytology in obtaining a histological diagnosis of unclear biliary strictures and alterations $[12$, $14,20]$.

First studies have shown the diagnostic value of dSOCs imaging for evaluating indeterminate biliary strictures and lesions as well a proposed classification for macroscopic characterization of determined stenosis. On the other hand, dSOC has been evaluated for guidewire placement into the region of interest or passing complex strictures and stenoses $[8,17,18]$.

Another important issue is the potential of dSOCs to reduce fluoroscopy time. As soon as the cholangioscope has been inserted in the biliary system, fluoroscopy is almost not needed any more. It already had been discussed in an Editorial if we can abandon fluoroscopy in the future [21]. We still relay on fluoroscopy during dSOC; however, the fluoroscopy time is markedly reduced using
dSOC (data were not evaluated in this retrospective study).

In our large study with 180 investigations in 117 patients, we confirm the procedural safety profile of dSOC procedures. No major complication occurred. The most common complications were cholangitis in $10 \%$ and mild bleeding in $<3 \%$ of the patients. The potential risk of cholangitis can further be reduced by applying peri-interventional antibiotic prophylaxis in all patients. Antibiotic prophylaxis has not been applied in our patient cohort in a standardized manner, but only in those patients with other reasons (preexisting cholangitis and dilation). However, our complication rate was rather low compared to earlier studies [12], even in our study population being rather ill, with high comorbidities or under immunosuppressive therapy in some cases. Unfortunately, our data do not allow to compare the risk of cholangitis in the population with or without antibiotic treatment. However, as procedural result of the study, we changed our protocol and perform peri-interventional antibiotic prophylaxis in all patients undergoing dSOC.

Almost all ROIs in the middle or upper CBD and the primary and secondary intrahepatic, ducts were successfully reached in our cohort. Biopsies were obtained if necessary. However, we clearly show that for the distal CBD, dSOC is much less effective in terms of visualization abil- 
ity of the ROI, which was successful in only two-thirds of the patients. Due to the instable position in the distal CBD, biopsies were even more difficult to obtain in those patients.

In cases with difficult cannulation, cholangioscopicguided guidewire cannulation is a proper option. This was efficient in all patients were standard guidewire cannulation failed repeatedly under fluoroscopic guidance.

A main focus of our study was to evaluate the diagnostic accuracy of the dSOCs imaging and visualization of biliary lesions and indeterminate strictures. The endoscopic imaging and macroscopic characterization of the investigator were compared with histology (or surgery) and follow-up of the patients. This is a remarkable strength of our study that a final diagnosis was obtained and therefore positive and NPV could be calculated adequately. We found an extremely high NPV of 0.97 in the whole study cohort. Therefore, the visual criteria to rule out a malignancy can be gathered very well by $\mathrm{dSOC}$, at least by experienced investigators. In focus of the clinical reality with additional imaging modalities and patients' history, we defined different clinical scenarios with different a-priori likelihood of an expected malignant stenosis. In patients with high a-priori likelihood, dSOC with macroscopic characterization of undetermined strictures revealed a very high test accuracy. In those patients with any biliary lesion ("high a-prioriy suspiscion" and "a-priori uncertainty"), sensitivity and specificity were 1.0 (95\% CI $0.86-1.0)$ and 0.76 (95\% CI $0.56-0.9)$ with a calculated PPV 0.77 (95\% CI 0.59-0.9) and a high NPV of 1.0 (95\% CI 0.85-1.0) for the visual diagnosis of biliary malignancy.

Even in cases with negative biopsies, these results encourage the use of dSOC in the diagnostic and management of biliary malignancy. Especially, in case of borderline resectable tumors with expected large surgical extend and associated mortality, dSOC enables endoscopists and surgeons to ensure the clinical management when discussing demanding surgical resection. In patients with a "borderline" a-priori likelihood, the NPV is still very high to ensure a further follow-up of an indeterminate biliary strictures.

The PPV with 0.77 to define a malignant transformation within the biliary system is higher than histology or brush cytology [14] but still needs to be improved. Most common benign strictures that were misdiagnosed as malignant were inflammatory strictures. Another advantage of dSOC is that the expansion of biliary lesions can much better be evaluated than in the fluoroscopy. Intramucosal changes are not apparent using X-ray only.
However, the more important point, in clinical practice when discussing the potential malignant pathogenesis of a biliary lesion or stenosis, is a reliable high NPV of dSOCbased visualization that helps for decision-making and leading the patients' further therapeutic options with more confidence.

Taken together, dSOC is a highly efficient method to evaluate indeterminate biliary lesions, stenoses, and strictures. In determinant biliary stenosis, accuracy of digital endoscopic biliary imaging by dSOC is quite high, with a remarkable NPV. If this finding is reproducible, dSOC possibly will be included in a standardized diagnostic and therapeutic procedure in patients with biliary lesions suspected for cholangiocarcinoma. Prospective, multicenter studies to confirm these data are already recruiting patients. In addition, our data confirm the high safety and technical efficacy of this new method. We believe adding dSOC in most ERCP procedures will become standard of care in the near future.

\section{Statement of Ethics}

This study was conducted in accordance with the Declaration of Helsinki and was approved by the Institutional Review Board of the University Hospital Regensburg. All interventions were routine procedures, and patients gave written informed consent prior to the procedure.

\section{Conflict of Interest Statement}

The authors declare that they have no conflicts of interest to disclose.

\section{Funding Sources}

No funding was received regarding this study.

\section{Author Contributions}

K.W. and A.K. conceptualized the study. Data curation was performed by K.W., M.K., A.K., and I.Z.J. The formal analysis was done by K.W., M.K., and A.K. No external funding was acquired. This study was supported by M.M.S. Investigation was done by K.W., I.Z.J., and A.K. K.W. and A.K. are responsible for project administration and methodology. Resources and supervision were given by M.M.S. Standard software was used. The original draft was written by K.W. and A.K. All authors validated, reviewed, and edited the manuscript. 


\section{References}

1 Paranandi B, Oppong KW. Biliary strictures: endoscopic assessment and management. Frontline Gastroenterol. 2017;8(2):133-7.

2 Cotton PB. Endoscopic retrograde cholangiopancreatography: maximizing benefits and minimizing risks. Gastrointest Endosc Clin N Am. 2012;22(3):587-99.

3 de Groen PC, Gores GJ, LaRusso NF, Gunderson LL, Nagorney DM. Biliary tract cancers. N Engl J Med. 1999;341(18):1368-78.

4 Singh A, Gelrud A, Agarwal B. Biliary strictures: diagnostic considerations and approach. Gastroenterol Rep. 2015;3(1):22-31.

5 Verdonk RC, Buis CI, Porte RJ, van der Jagt EJ, Limburg AJ, van den Berg AP, et al. Anastomotic biliary strictures after liver transplantation: causes and consequences. Liver Transpl. 2006;12(5):726-35.

6 Yasuda I, Itoi T. Recent advances in endoscopic management of difficult bile duct stones. Dig Endosc. 2013;25(4):376-85.

7 Fritcher EG, Kipp BR, Halling KC, Oberg TN, Bryant SC, Tarrell RF, et al. A multivariable model using advanced cytologic methods for the evaluation of indeterminate pancreatobiliary strictures. Gastroenterology. 2009; 136(7):2180-6.

8 Tieu AH, Kumbhari V, Jakhete N, Onyimba F, Patel Y, Shin EJ, et al. Diagnostic and therapeutic utility of SpyGlass ${ }^{\circ}$ peroral cholangioscopy in intraductal biliary disease: singlecenter, retrospective, cohort study. Dig Endosc. 2015;27:479-85.
9 Chen YK. Preclinical characterization of the SpyGlass peroral cholangiopancreatoscopy system for direct access, visualization, and biopsy. Gastrointest Endosc. 2007;65(2):30311.

10 Chen YK, Pleskow DK. SpyGlass single-operator peroral cholangiopancreatoscopy system for the diagnosis and therapy of bile-duct disorders: a clinical feasibility study (with video). Gastrointest Endosc. 2007;65(6):832-41.

11 Laleman W, Verraes K, Van Steenbergen W, Cassiman D, Nevens F, Van der Merwe S, et al. Usefulness of the single-operator cholangioscopy system SpyGlass in biliary disease: a single-center prospective cohort study and aggregated review. Surg Endosc. 2017;31(5): 2223-32.

12 Lenze F, Bokemeyer A, Gross D, Nowacki T, Bettenworth D, Ullerich H. Safety, diagnostic accuracy and therapeutic efficacy of digital single-operator cholangioscopy. United European Gastroenterol J. 2018;6(6):902-9.

13 Shah RJ, Raijman I, Brauer B, Gumustop B, Pleskow DK. Performance of a fully disposable, digital, single-operator cholangiopancreatoscope. Endoscopy. 2017;49(7):651-8.

14 Rey JW, Hansen T, Dümcke S, Tresch A, Kramer K, Galle PR, et al. Efficacy of SpyGlass ${ }^{\mathrm{m}}$-directed biopsy compared to brush cytology in obtaining adequate tissue for diagnosis in patients with biliary strictures. World J Gastrointest Endosc. 2014; 6(4):137-43.

15 Woo YS, Lee JK, Oh SH, Kim MJ, Jung JG, Lee $\mathrm{KH}$, et al. Role of SpyGlas peroral cholangioscopy in the evaluation of indeterminate biliary lesions. Dig Dis Sci. 2014;59(10):2565-70.
16 Brewer Gutierrez OI, Bekkali NLH, Raijman I, Sturgess R, Sejpal DV, Aridi HD, et al. Efficacy and safety of digital single-operator cholangioscopy for difficult biliary stones. Clin Gastroenterol Hepatol. 2018;16(6):918e1.

17 Bokemeyer A, Gross D, Bruckner M, Nowacki T, Bettenworth D, Schmidt H, et al. Digital single-operator cholangioscopy: a useful tool for selective guidewire placements across complex biliary strictures. Surg Endosc. 2019 Mar;33(3):731-7.

18 Husing-Kabar A, Heinzow HS, Schmidt HH, Stenger C, Gerth HU, Pohlen M, et al. Singleoperator cholangioscopy for biliary complications in liver transplant recipients. World J Gastroenterol. 2017;23:4064-71.

19 Robles-Medranda C, Valero M, Soria-Alcivar M, Puga-Tejada M, Oleas R, Ospina-Arboleda J, et al. Reliability and accuracy of a novel classification system using peroral cholangioscopy for the diagnosis of bile duct lesions. Endoscopy. 2018;50(11):1059-70.

20 Bernica J, Elhanafi S, Kalakota N, Jia Y, Dodoo C, Dwivedi A, et al. Cholangioscopy is safe and feasible in elderly patients. Clin Gastroenterol Hepatol. 2018;16(8):1293-e2.

21 Raijman I. Performing endoscopic retrograde cholangiography without radiation exposure: are we ready for it? Gastrointest Endosc. 2016; 84(5):770-2. 